\title{
TAXAS DE TRANSPORTE SELETIVO PARA DIFERENTES TAMANHOS DE PARTÍCULAS AO LONGO DE UMA PRAIA: REPERCUSSÃO NO REGISTRO SEDIMENTAR
}

\author{
ABÍLIO C. S. P. BITTENCOURT*, GERALDO S. V. BOAS*, \\ ANTÓNIO E. G. AZEVEDO* e FÉLIX F. FARIAS***
}

\begin{abstract}
SELECTIVE LONGSHORE GRAIN SIZE TRANSPORT RATES: REPERCUSSION ON THE SEDIMENTARY RECORD. Monitoring of two beaches profiles along the Caixa-Pregos spit (Todos os Santos Bay, Bahia) extending in time from June/1987 to August/1988 has shown that coarse, medium and very fine sands move faster than fine sand under the action of the longshore drift. This selective longshore transport is related to differences in hyclrodynamic behavior of the various sand size fractions. Coarse and medium sand move continuously as bedload on the beach face, whereas very fine sand goes in suspension. Fine sand lags behind probably because it is transported in saltation. Because the construction phase at the Caixa-Pregos split temporally coincides with an increase in the longshore component of the wave energy flux, thus enhancing the selective grain size transport, such phase if preserved in the stratigraphic record, should be characterized by a relative increase in the beach-face deposits, of the coarse, medium and very fine sand fractions in comparision to the fine sand fraction. These grain size trends however, have not been observed at the Caixa-Pregos spit deposits, because the erosion of these sediments during the subsequent destructional phase of the beach cycle. The preservation in the stratigraphic record of the grain size trends reported herein requires a positive balance between constructional and destructional phases of beach cycle, thus resulting in progradation of the shoreline. In addition it is also required that, during the constructive phase there is a considerable reduction in the frequency of small erosional events, that affects the beach-face. Such scenario will be feasible in this low latitude sand spit if for example, the periodic advances in the Polar Atlantic Front, which cause true erosional $\mathrm{S}$ and SSE winds, are reduced by some atmospheric barrier like the El Niño phenomenon. The effectiveness of such phenomenon in controlling longhshore sediment transport has already been demonstrated for the Armação Beach (Bahia).
\end{abstract}

Keywords: Beach sedimentation dynamics, longshore drift, grain-size selective transport.

RESUMO O monitoramento de dois perfis praiais localizados nas extremidades do esporão de Caixa-Pregos (Baía de Todos os Santos, Bahia), durante o período de junho/1987 a agosto/1988, mostrou que as frações granulométricas de areia grossa, média e muito fina movimentam-se, pela ação da deriva litorânea, com maiores velocidades do que a areia fina. Este transporte seletivo ao longo da praia está relacionado às diferenças no comportamento hidrodinâmico dos vários tamanhos de grão. Na face da praia, as areias grossa e média movem-se continuamente na carga de fundo, enquanto a areia muito fina, em suspensão. A areia fina fica em atraso provavelmente porque ela é transportada por saltação. Devido ao fato de a fase construtiva no esporão de Caixa-Pregos coincidir temporalmente com um aumento da compomente, ao longo da praia, do fluxo de energia das ondas, dessa maneira aumentando o transporte seletivo de grãos, tal fase, se preservada no registro estratigráfico, será caracterizada por um aumento relativo, nos depósitos da face da praia, das frações granulométricas de areia grossa, média e muito fina, em comparação com a areia fina. Essa sequência granulométrica não foi observada nos depósitos do esporão de Caixa-Pregos, devido à erosão da mesma durante a fase destrutiva subsequente do ciclo praial. A preservação no registro estratigráfico de tal sequência requer um balanço positivo entre as fases construtivas e destrutivas do ciclo praial, resultando numa progradação da linha da costa. Ademais, será também necessário que, durante a fase construtiva, exista uma considerável redução na frequência dos pequenos eventos erosivos que afetam a face da praia. Esse cenário será viável neste esporão de baixa latitude se, por exemplo, os periódicos avanços da Frente Polar Atlântica, que provocam os ventos de S e de SSE, francamente erosivos, forem reduzidos por alguma barreira atmosférica como o fenómeno El Niño. A efetividade desse fenômeno em controlar o transporte de sedimentos ao longo da praia foi demonstrado na praia de Armação (Bahia).

INTRODUCÃO Palavras-chaves: Dinâmica de sedimentação praial, deriva tudos experimentais, chegaram à conclusão de que, desde que o transporte, sob a ação da deriva litorânea, seja feito na carga de fundo, quanto maior o tamanho do grão de areia, maior a velocidade com que o mesmo é transportado ao longo da praia. Komar (1977) observou que, no caso de transporte feito por suspensão, a tendência é inversa, ou seja, as partículas tendem a ter velocidades maiores quanto menores forem os seus tamanhos. Esse mesmo autor conseguiu traçar uma curva que relaciona a velocidade da partícula com a granulometria, a partir de dados colhidos na praia de El Moreno, no México. Recentemente, Bittencourt et al (1990) realizaram um trabalho de monitoramento das variações temporais e espaciais das praias localizadas ao longo do esporão de Caixa-Pregos, torânea, transporte seletivo de grãos.

na extremidade sul da ilha de Itaparica, na entrada da Baía de Todos os Santos (Fig. 1); os mesmos definiram o mecanismo de construção dessa feição geomórfica, que está associado essencialmente à deriva litorânea. Durante o período de 25 de junho/1987 a 22 de dezembro/1988, Bittencourt et al (1990) identificaram nas praias localizadas ao longo do corpo do esporão uma fase construtiva seguida de uma destrutiva, ambas muito bem marcadas, e de uma outra ligeiramente construtiva (Figs. 2B e 2C). Segundo esses autores, as fases construtivas estão relacionadas à predominância de ventos providos de NE, E e SE (Fig. 2A), que promovem uma deriva litorânea para o esporão, a partir das praias localizadas a leste do mesmo, neste trabalho denominadas fonte (Fig. 3A). A fase destrutiva está relacionada à existência de ventos prove-

\footnotetext{
* Programa de Pesquisa Pós-Graduação em Geofísica, Instituto de Geociências, Universidade Federal da Bahia, Rua Caetano Moura, 123, Campus Universitário, Federação, CEP 40210, Salvador, BA, Brasil
} 


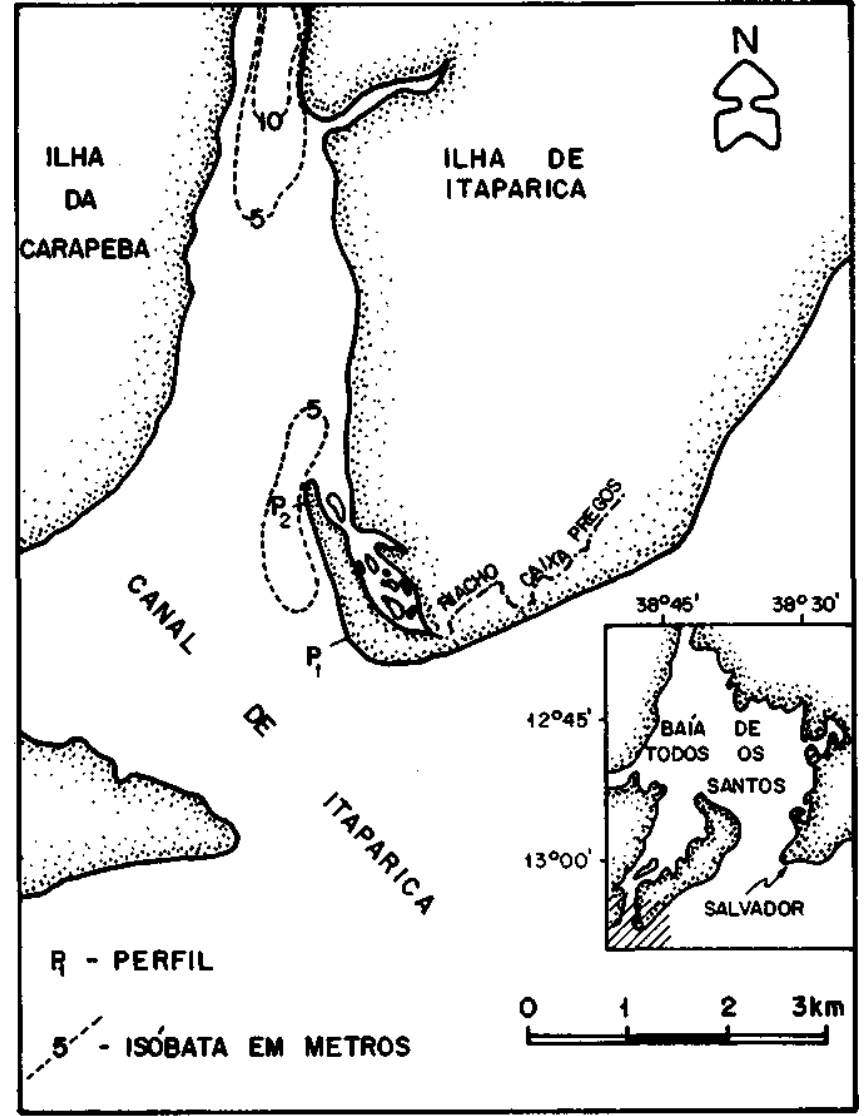

Figura 1 - Mapa de localização do esporão de Caixa-Pregos mostrando o posicionamento dos perfis (modificado de Bittencourt et al. 1990)

Figure 1 - Location of the Caixa-Pregos spit with studied transects (modified from Bittencourt et al. 1990)

nientes de SSE e de S (Fig. 2A), francamente erosivos (Figs. $3 \mathrm{~B}$ e $3 \mathrm{C}$ ). Atualmente, segundo esses autores, os sedimentos trazidos da fonte pela deriva litorânea não mais se acumulam na ponta do esporão, tendo o mesmo parado de crescer. Ao invés disso, pela ação das correntes de vazante, estão sendo lançados, a partir da ponta do esporão, num canal contíguo ao mesmo. Bittencourt et al (1990) também chegaram à conclusão de que, ao longo do esporão, são desprezíveis as movimentações de materiais pela ação das ondas, no sentido da antepraia para a face da praia e vice-versa, bem como da face da praia para o pós-praia, pela ação dos ventos.

O esporão de Caixa-Pregos, por apresentar uma movimentação de materiais praticamente apenas no sentido longitudinal das praias, sem ponderáveis deslocamentos transversais, reúne características ambientais apropriadas para se estudar, durante fases erosivas e construtivas, a repercurssão do processo de transporte seletivo de partículas pela deriva litorânea na sedimentação praial, o que é o objetivo do presente trabalho. Para alcançar tal objetivo, foram selecionadas apenas as amostras do sedimento praial coletadas por Bittencourt et al (1990) nas duas primeiras fases do monitoramento, abrangendo o período de junho/1987 a agosto/1988, uma vez que, como mencionado anteriormente, durante a terceira fase houve pouca movimentação de materiais, estando o seu caráter construtivo levemente manifesto nos gráficos. Essas amostras, em número de 25 , coletadas na face da praia superior dos perfis praiais $\mathrm{P} 1 \mathrm{e}$ P2, próximos ao início e ao fim do esporão, respectivamente, são representativas dos primeiros $2 \mathrm{~cm}$ superficiais do sedimento praial (Fig. 1). Após secada e quarteada, de cada amostra

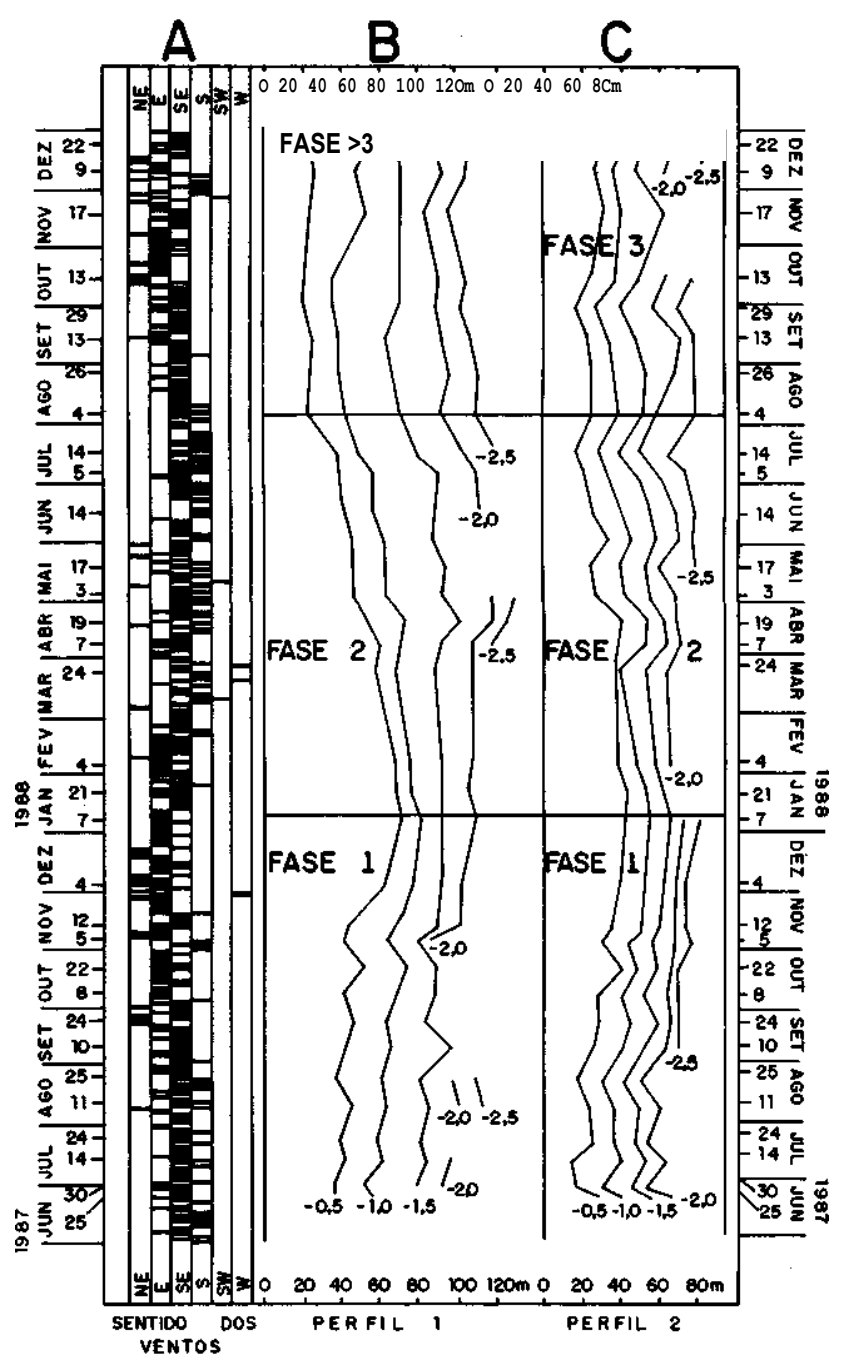

Figura 2 - A. sentido dos ventos na Baía de Todos os Santos; $B$ e C. diagramas de linhas de contorno para os perfis l e 2 , respectivamente. Os contornos ligam pontos de cotas iguais na superficie da praia, em metros, medidas ao longo do tempo em relação a um datum que passa pelo pós-praia (modificado de Bittencourt et al. 1990)

Figure 2 - A. wind direction in Todos os Santos Bay; B and $\mathbf{C}$. isopleth diagrams showing variations in bcach surface elevation through time measured from a horizontal datum that intercepts the beach prism in the backshore. Lines connect equal elevation points relative to this datum. Diagrams depici variations on profiles 1 and 2 (modified from Bittencourt et al 1990)

foi retirada uma quantidade em torno de $50 \mathrm{~g}$ para a eliminação dos sais solúveis. Por fim, a amostra foi peneirada a seco com Rotap, através de um conjunto de peneiras com intervalos de $1 / 2 \Phi$

ANÁLISE COMPARATIVA DA DISTRIBUIČ̃̃O GRANULOMETRICA DO SEDIMENTO PRAIAL DOS PERFIS P1 E P2 COLETADO DURANTE O PERÍODO DE JUNHO1987 A AGOSTO/1988 De uma maneira geral, ao longo do tempo, os perfis PI e P2, granulometricamente, apresentam um comportamento bastante assemelhado entre si. Na fase construtiva, nos dois perfis, observa-se a existência de uma tendência, do início para o fím da fase, de um aumento nas porcentagens de areias média $(0,500-0,250 \mathrm{~mm})$ e muito fina $(0,125-0,062 \mathrm{~mm})$; em contrapartida, há uma diminuição nas porcentagens de areia fina $(0,250-0,125 \mathrm{~mm})$ (Figs. $4 \mathrm{e}$ 

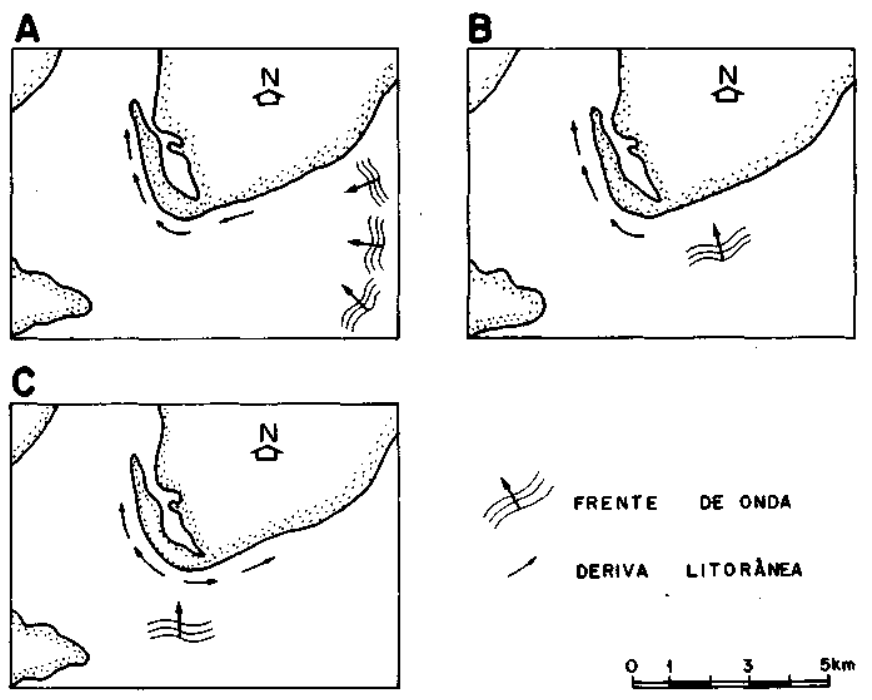

Figura 3 - Mapas esquemáticos dos padrões de deriva litorânea gerados por ventos provindos de nordeste, leste e sudeste (A), sul-sudeste (B) e sul (C) (Bittencourt et al. 1990) Figure 3 - Schematic charts showing the longshore drift of sedimenta induced by winds from NE, E and SE (A), SSE (B) and S (C) (Bittencourt et al. 1990)

5). É notável que, apenas próximo ao final da fase construtiva, aparece a fração areia grossa $(1,00$ - 0,500) nos dois perfis, embora em porcentagens muito pequenas. Em relação à fase erosiva, constata-se que as porcentagens de areias fina e muito fina nos dois perfis, inversamente ao observado na fase construtiva, denotam tendências, respectivamente, para crescimento e diminuição, do início para o fim da fase (Figs. 4 e 5). Sobre as variações percentuais de areia média, não existe, ao longo do tempo, uma tendência definida nessa fase. Porém as porcentagens são marcadamente inferiores às da fase construtiva (Figs. 4 e 5).

DISCUSSÃO E CONCLUSÕES O peculiar comportamento, ao longo do tempo, das variações percentuais das diferentes frações granulométricas presentes nas praias do esporão de Caixa-Pregos é entendido, fundamentalmente, como uma decorrência do transporte seletivo de partículas pela ação da deriva litorânea. Como já apontado, movimentações transversais de materiais nessas praias não são significativas.

Numa praia em que as partículas, sob a ação da deriva litorânea, são transportadas com diferentes velocidades, e em que a mesma está em processo de construção, as sucessivas lâminas de sedimentos que vão se sobrepondo ao longo do tempo devem apresentar maiores porcentagens daquelas partículas que, a par de terem maiores velocidades de transporte, representam uma fração significativa do total da distribuição granulométrica na fonte. Do contrário, como aponta Komar (1977), elas não serão importantes para o volume total de areia transportado. Isto quer dizer que, mesmo que uma partícula tenha uma alta velocidade de transporte, se ela representa apenas um ínfimo percentual no total da distribuição granulométrica na fonte, o resultado final é que ela será sobrepujada durante o transporte, em volume, por uma outra mais lenta que, todavia, represente um considerável percentual na fonte. No caso de Caixa-Pregos, duas amostras coletadas na fonte por T.M.F. Araújo e analisadas por estes autores mostraram que as partículas da fração areia fina são sempre porcentualmente bem mais elevadas do que as frações areias muito fina, média e grossa - a última com porcentagens bem reduzidas. Desse modo, o fato de as porcentagens de areia fina na fase construtiva, por exemplo, diminuirem à medida que aumentam as porcentagens das areias média e muito fina (Figs. 4 e 5), só pode ser entendido quando se considera que a areia fina se desloca com menor velocidade do que as outras duas frações. Do contrário, a tendência seria inversa. Segundo esse modelo, as sucessivas lâminas sobrepostas durante a fase construtiva, caso as diferenças de velocidades de transporte entre as partículas se mantiverem constantes ao longo do tempo e não haja mudanças na composição da fonte, deverão apresentar sempre as mesmas diferenças percentuais entre as frações granulométricas presentes no sedimento.

A figura 6 mostra, em linha cheia, a curva resultante da velocidade de transporte da partícula versus a granulometria traçada por Komar (1977) para a praia de El Moreno. Nessa mesma figura aparecem, em linhas tracejadas, outras curvas aproximadas traçadas pelo mesmo autor para outras situações de onda e de deriva litorânea diferentes das encontradas no México. Basicamente, essas outras curvas são paralelas à traçada para a praia de El Moreno, diferençando-se apenas o seu posicionamento no gráfico: são deslocadas para cima ou para baixo, a depender se as condições das ondas produzem, respectivamente, maiores ou menores velocidades de transporte. Por esse gráfico, é possível observar ainda que, para dois determinados tamanhos de partículas, quanto maior for a intensidade da componente ao longo da praia do fluxo de energia das ondas, tanto maior será a diferença entre as velocidades de transporte das mesmas. Nas praias do esporão de Caixa-Pregos, é possível observar que as velocidades com que as frações granulométricas presentes no sedimento praial se deslocam ao longo da praia estão em desacordo com o encontrado por Komar (1977) na praia de El Moreno. Pela figura 6, constata-se que, na praia de El Moreno, a fração de areia média, de uma maneira geral, apresenta menor velocidade de transporte do que as frações de areia fina e muito fina. Para as condições de Caixa-Pregos, os dados do presente trabalho sugerem que a curva de Komar (1977) (Fig. 6) deve ser deslocada para a direita do gráfico, de tal forma que o ponto da curva correspondente à menor velocidade de transporte posicione-se aproximadamente no meio da faixa correspondente à areia fina. Nessa situação, as areias média e fina, bem como a areia grossa, têm uma maior velocidade de transporte do que a areia fina, situação que, conforme mencionado anteriormente, é encontrada em Caixa-Pregos.

A discrepância entre os dados das praias de El Moreno e de Caixa-Pregos deve estar relacionada às diferentes condições de onda existentes nas mesmas. El Moreno é uma praia que apresenta uma intensa zona de espraiamento (Komar 1977), marcadamente diferente de Caixa-Pregos, que é, conforme já visto, uma praia com baixa energia. Segundo Komar (1977), o ponto de sua curva, relativo às partículas que se movimentam com menores velocidades de transporte, deve corresponder (Fig. 6) àquele que separa o mecanismo de transporte por carga de fundo, no sentido das frações mais grossas, do por suspensão, no sentido das mais finas. Assim é que a fração de areia fina - na praia de El Moreno, com maior energia, transportada francamente por suspensão e, portanto, com maior velocidade do que a areia média, em Caixa-Pregos, com baixa energia - passa agora a manter contato com o fundo e a ser transportada com menor velocidade do que a areia média, que passa a ser transportada francamente na carga de fundo. A areia muito fina, mantendo-se no transporte em suspensão, toma-se também mais veloz do que a areia fina.

O regime de ventos responsável pela deriva litorânea ao longo do tempo de monitoramento na região de Caixa-Pregos é mostrado na figura 2A. Por essa figura, constata-se que na fase construtiva há uma passagem, do início para o fim da mesma, de uma faixa de tempo em que há predominância de ventos provindos de SE para outra em que prevalecem ventos provenientes de E. Essa mudança no sentido dos 


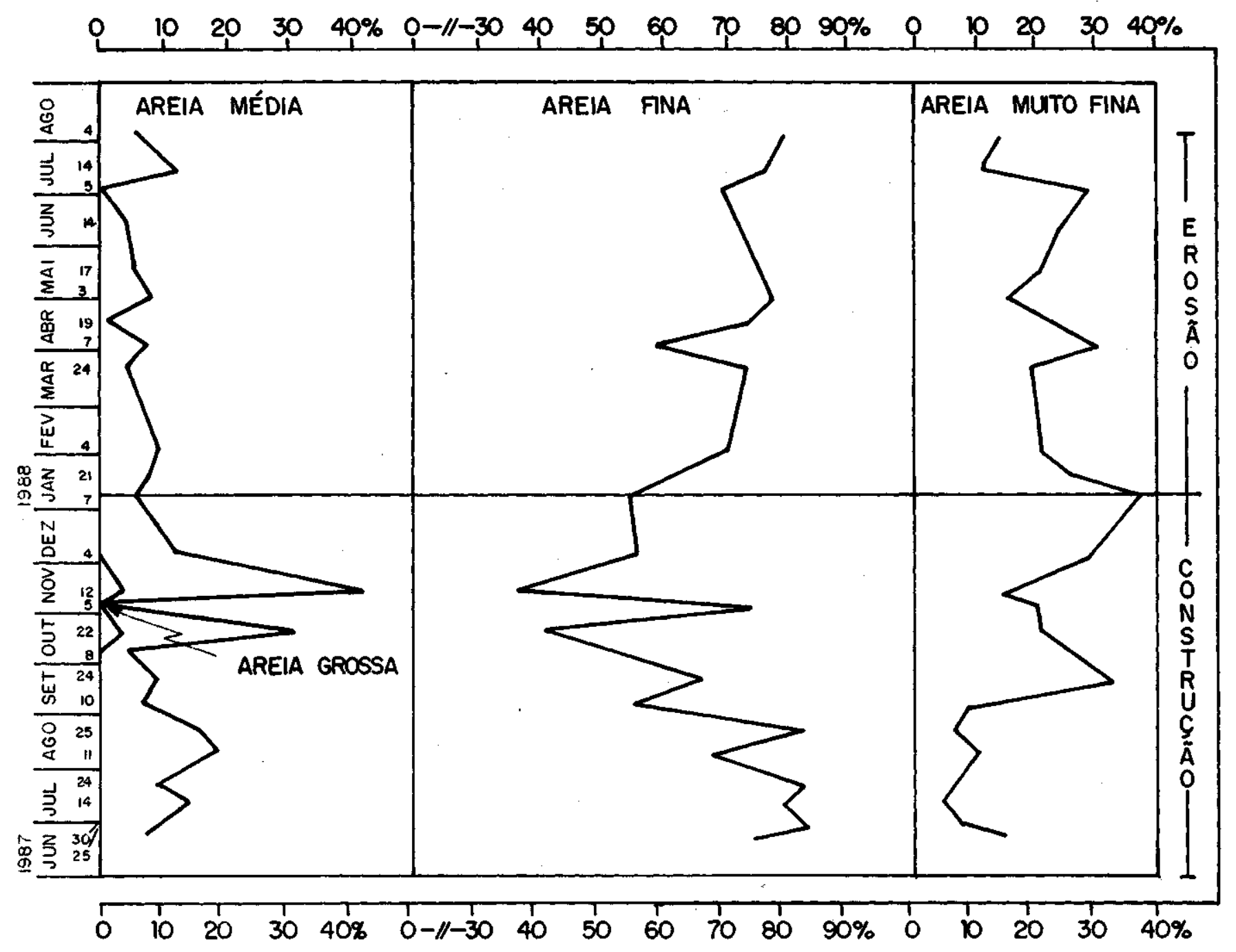

Figura 4 - Variações nas porcentagens das frações granulométricas de areia grossa, média, fina e muito fina no perfil P1, ao longo do tempo

Figure 4 - Percentual changes in the grain size proportion of coarse, médium, fine and very fine sands at profile P1 during the time

ventos vai implicar no aumento da intensidade da componente ao longo da praia do fluxo de energia das ondas, que, segundo Komar (1977), redundará num incremento na diferença entre as velocidades de transporte das partículas. Assim, pelos gráficos que mostram as fases de erosão e construção dos perfis P1 e P2, nota-se que existe, na fase construtiva sob apreciação neste trabalho, notadarnente no perfil PI (Fig. 2B), urna estreita relação entre a velocidade de construção do perfil praial e o regime de ventos. Nesse sentido, a velocidade de crescimento é maior no período de tempo em que predominam ventos de E, devido ao fato deles provocarem uma deriva litorânea com intensidade maior do que aquela causada pelos ventos de SE. Na fase erosiva, em relação aos ventos que são responsáveis por tal processo, provenientes de SSE e de S (Fig. 2A) (Bittencourt et al. 1990), há $u$ ma faixa de tempo, no início da fase, em que os primeiros (na Fig. 2A assinalados simplesmente como do quadrante SE) aparecem praticamente desacompanhados de ventos de $\mathrm{S}$ e, uma segunda, no restante da fase, em que ocorrem com frequências assemelhadas aos ventos de S. De forma semelhante à fase construtiva, tal distribuição no regime de ventos vai também provocar, do início para o fim da fase, um aumento na intensidade da componente ao longo da praia do fluxo de energia das ondas e, conseqüentemente, da mesma forma, um incremento na diferença entre as velocidades de transporte das partículas.

Pelas figuras $2 \mathrm{~B}$ e $2 \mathrm{C}$, o período de tempo em que o perfil praial erode mais rapidamente coincide exatamente com aquele em que ocorrem os ventos de $\mathrm{S}$, os quais promovem uma deriva litorânea mais intensa do que os ventos de SSE.

No caso das praias do esporão de Caixa-Pregos, durante a fase construtiva, já que existe um aumento entre as diferenças de velocidade de transporte das partículas com o tempo, as sucessivas lâminas que vão se sobrepondo vão apresentar crescentes aumentos nas diferenças percentuais entre as partículas mais velozes e menos velozes, respectivamente - como já apontado, areias média e muito fina e areia fina (Figs. 4 e 5). Na parte final da fase construtiva, quando são maiores essas diferenças de velocidade, a fração de areia grossa - a qual, segundo Komar (1977), apresenta uma maior velocidade de transporte do que as frações de areia média, fina e muito fina - consegue ter, apesar de na fonte apresentar porcentagens muito baixas, uma expressão percentual nos gráficos das figuras 4 e 5. Quando cessa a alimentação de sedimentos para o esporão, durante a fase erosiva, as lâminas de sedimentos 


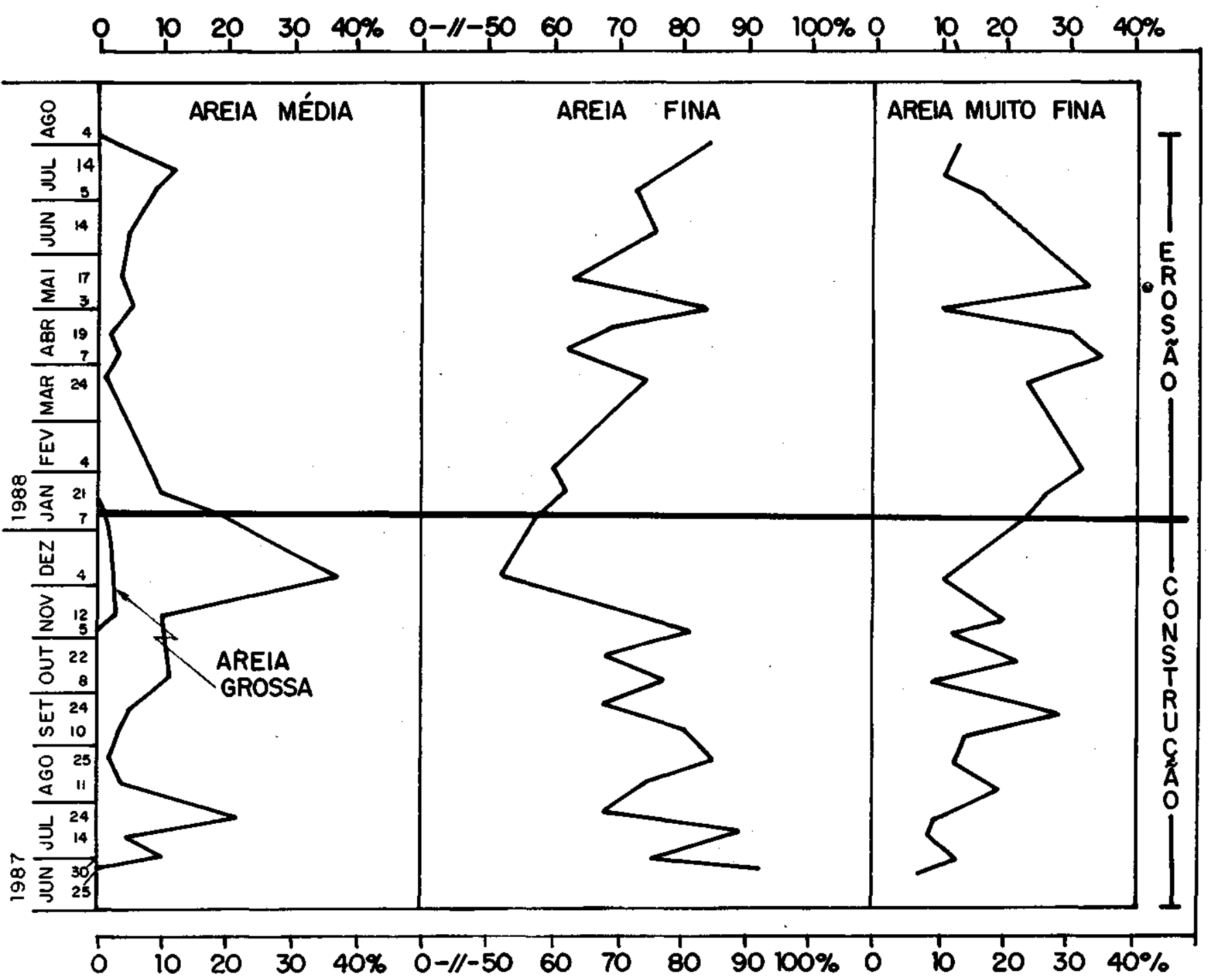

Figura 5 - Variações nas porcentagens das frações granulométricas de areia grossa, média, fina e muito fina no perfil P2, ao longo do tempo

Figure 5 - Percentual changes in the grain size proportion of coarse, médium, fine and very fine sands at profile P2 during the time

depositadas na fase construtiva antecedente vão sendo sucessivamente exumadas ao longo do tempo. Dessa maneira, deverão ser expostas, gradativamente, camadas que contenham, cada vez em porcentagens menores, as frações que foram depositadas em volumes crescentes na fase construtiva anterior. Nas praias do esporão de Caixa-Pregos, essa tendência deverá ser acentuada, já que, na fase erosiva, as diferenças de velocidade de transporte entre as partículas aumentam do inicio para o fim da fase. Assim, as partículas que na fase construtiva foram aumentando porcentualmente ao longo do tempo por serem transportadas com cada vez maiores velocidades em relação às mais lentas, como uma resposta a um aumento entre as diferenças de velocidade das mesmas, serão erodidas na fase erosiva, ao longo do tempo, pela mesma razão, em quantidades cada vez maiores.

Na realidade, esse modelo não é constatado nos gráficos das figuras 4 e 5, pois, em Caixa-Pregos, as fases construtivas apresentam, de tempos em tempos, pequenas fases erosivas (Figs. 2B e 2C). Desse modo, por exemplo, ao se passar por uma pequena fase erosiva no perfil P1, do dia 22/10/87 a 05/11/87 (Fig. 2B), a areia média, que estava com uma porcentagem relativamente alta, é rapidamente erodida pelos ventos de $\mathrm{S}$ (Fig. 2A) e passa a ficar com uma porcentagem muito baixa (Fig. 4). Isso significa que as diferentes porcentagens das frações granulométricas presentes no sedimento praial, monitoradas na fase construtiva, não permanecem necessariamente no regime estratigráfico. Assim, na fase erosiva, tanto no perfil P1quanto no perfil P2, não se observam, em relação à fração areia média, o que seria previsível segundo o modelo acima descrito. Apenas o gráfico das porcentagens de areia muito fina, nos dois perfis, sugere uma ligeira tendência de diminuição dos valores com o tempo (Figs. 4 e 5). De forma relativa, isso implica numa tendência inversa em relação à areia fina, já que a areia média não apresenta variações significativas. Esse fato pode estar relacionado a aportes de areia muito fina, durante a fase construtiva, a partir da antepraia para a face da praia, nas praias localizadas a leste do esporão. O aporte extra, aumentando a porcentagem de areia muito fina na fonte, faria aumentar a quantidade de materiais transportados da mesma, o que compensaria as perdas, durante as pequenas fases erosivas, que ocorreram durante a fase construtiva. Devido a esse fato é que, durante a fase erosiva, o comportamento das variações percentuais da areia muito fina, 


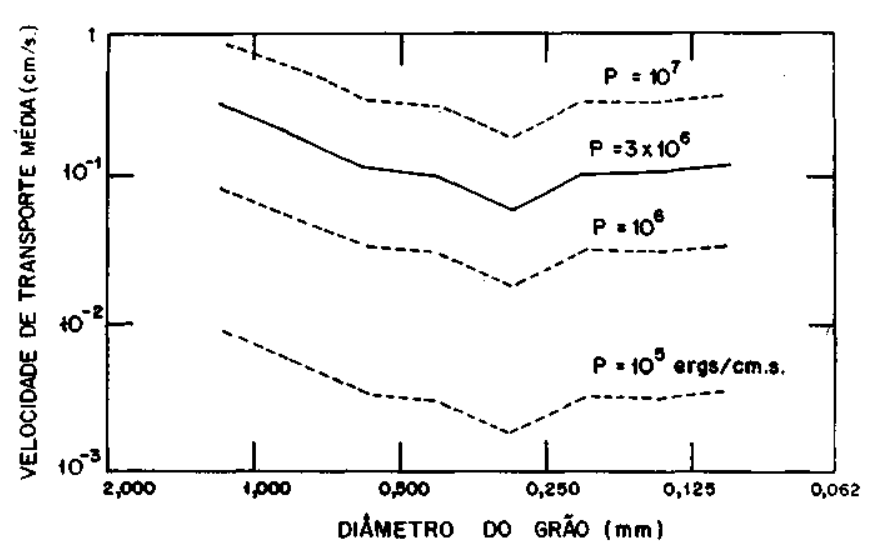

Figuro 6 - Velocidades de transporte seletivo médias ao longo da praia para diferentes frações granulométricas de areia da praia de El Moreno (México) em função das condições das ondas. Este nível, denominado de $P$, representa a componente, ao longo da praia, do fluxo de energia das ondas. $A$ curva de $P=3 \times 10$ ergs $/ \mathrm{cm}$.s corresponde à praia de El Moreno. As demais outras não são diretamente baseadas em dados, sendo deslocadas para cima ou para baixo, considerando-se o aumento ou decréscimo do transporte total de areia em função das condições das ondas (modificado de Komar 1977)

Figure 6 - Mean selective longshore transport velocities for the different grain-size fraction of El Moreno Beach sand as a function of the levei of the wave conditions, denoted by $\mathrm{P}$, (the longshore component of the wave energy flux. The curve P - 3 x 10 ergs $/ \mathrm{cm} . s$ corresponde El Moreno Beach. The other curves are not directly based on data, being displaced upward or downward to accoimt for increasing or decreasing total transport as a function of the wave conditions (Modified from Komar 1977)

\section{REFERÊNCIAS BIBLIOGRÁFICAS}

BITTENCOURT, A.C.S.P.; FARIAS, F.F.; VILAS BOAS, G.S. 1990. Influência cia deriva litorânea no desenvolvimento do esporão de Caixa-Pregos (Baía de Todos os Santos/BA). Rev. Brás. Geoc., 20:197-200.

EVANS, Q.F. 1939. Sorting and transportation of material in the swash and backwash. Journ. Sed, Pet., 9:28-31.

FARIAS, F.F.; BITTENCOURT, A.C.S.P.; ZANINI A. Jr.; DOMINGUEZ, J.M.L. 1985. Variações temporais e espaciais na dinâmica de sedimentação da praia de Armação - Salvador/BA. Rev. Brás. Gcoc., 15:48-54.

KOMAR, P.D. 1977. Selective longshore transport rates of different grain-size fractions within a beach. Journ. Sed. Pet., 47:1444-1453. contrariamente ao da areia média, é compatível com o modelo anteriormente descrito.

Sobre a repercussão dos processos sedimentares já discutidos no registro sedimentar, a diferenciação percentual, ao longo do tempo, observada entre as frações granulométricas presentes num sedimento praial durante uma fase construtiva, só poderá ser preservadas quando o balanço entre as fases construtivas e erosivas for positivo, propiciando uma progradação da linha da costa. Ademais, é necessário ainda que, durante as fases construtivas, haja, senão o desaparecimento, mas uma considerável diminuição dos pequenos eventos erosivos que, de tempos em tempos, como foi constatado em Caixa-Pregos, atuam para destruir a sequência de aumento gradativo no registro estratigráfico das porcentagens das frações granulométricas que se deslocam com maiores velocidades sob o efeito da deriva litorânea. Essa circunstância só será possível, no caso de Caixa-Pregos, se porventura houver alguma barreira atmosférica que dificulte os avanços periódicos da Frente Polar Atlântica para latitudes mais baixas, a qual é a responsável, segundo a Secretaria de Planejamento, Ciência e Tecnologia do estado da Bahia - Seplantec (1978), pelos ventos provindos de SSE e de S, francamente erosivos. Tal fenómeno tem se manifestado efetivamente de tempos em tempos no Atlântico Sul, sob o nome de "El Niño" (Lagos 1981). O poder dessa barreira em inibir a chegada dos ventos de SSE e de S foi medido diretamente por Farias et al (1985) na praia de Armação, localizada na costa atlântica de Salvador, próximo a CaixaPregos, durante o ano de 1983.

Agradecimentos Os autores externam seus agradecimentos ao professor José Maria Landim Dominguez pelas críticas e sugestões apresentadas, bem como pela versão do resumo para o inglês.

LAGOS, P. 1981. "El Niño": alteraciones en la atmosfera y alerta en el mar. Rev. Geof., 14/15:5-12.

SECRETARIA DE PLANEJAMENTO, CIÊNCIA E TECNOLOGIA DO ESTADO DA BAHIA - SEPLANTEC 1978. Atlas Climatológico do Estado da Bahia, documento síntese. Salvador, Centro de Planejamento da Bahia (CEPLAB). 191p.

MANUSCRITO A686 Recebido em 02 de janeiro de 1991 Revisão do autor em de 01 de abril de 1991 Revisão aceita em 31 cie maio de 1991 